\title{
GABAergic Synapses at the Axon Initial Segment of Basolateral Amygdala Projection Neurons Modulate Fear Extinction
}

\author{
Rinki Saha', Stephanie Knapp ${ }^{2,3,4}$, Darpan Chakraborty', Omer Horovitz ${ }^{5}$, Anne Albrecht ${ }^{1,6}$, Martin Kriebel ${ }^{7}$, \\ Hanoch Kaphzan', Ingrid Ehrlich ${ }^{2,3}$, Hansjürgen Volkmer ${ }^{7}$ and Gal Richter-Levin ${ }^{*, 1,5,6}$ \\ 'Sagol Department of Neurobiology, University of Haifa, Haifa, Israel; ${ }^{2}$ Hertie Institute for Clinical Brain Research, University of Tübingen, Tübingen, \\ Germany; ${ }^{3}$ Centre for Integrative Neuroscience, University of Tübingen, Tübingen, Germany; ${ }^{4}$ Graduate School for Neural and Behavioral Science, \\ University of Tübingen, Tübingen, Germany; ${ }^{5}$ Department of Psychology, University of Haifa, Haifa, Israel; ${ }^{6}$ The Institute for the Study of Affective \\ Neuroscience, University of Haifa, Haifa, Israel; ${ }^{7} \mathrm{NMI}$ Natural and Medical Sciences Institute, University of Tübingen, Reutlingen, Germany
}

\begin{abstract}
Inhibitory synaptic transmission in the amygdala has a pivotal role in fear learning and its extinction. However, the local circuits formed by GABAergic inhibitory interneurons within the amygdala and their detailed function in shaping these behaviors are not well understood. Here we used lentiviral-mediated knockdown of the cell adhesion molecule neurofascin in the basolateral amygdala (BLA) to specifically remove inhibitory synapses at the axon initial segment (AIS) of BLA projection neurons. Quantitative analysis of GABAergic synapse markers and measurement of miniature inhibitory postsynaptic currents in BLA projection neurons after neurofascin knockdown ex vivo confirmed the loss of GABAergic input. We then studied the impact of this manipulation on anxiety-like behavior and auditory cued fear conditioning and its extinction as BLA related behavioral paradigms, as well as on long-term potentiation (LTP) in the ventral subiculumBLA pathway in vivo. BLA knockdown of neurofascin impaired ventral subiculum-BLA-LTP. While this manipulation did not affect anxietylike behavior and fear memory acquisition and consolidation, it specifically impaired extinction. Our findings indicate that modification of inhibitory synapses at the AIS of BLA projection neurons is sufficient to selectively impair extinction behavior. A better understanding of the role of distinct GABAergic synapses may provide novel and more specific targets for therapeutic interventions in extinction-based therapies.
\end{abstract}

Neuropsychopharmacology (2017) 42, 473-484; doi: I 0.1038/npp.2016.205; published online 19 October 2016

\section{INTRODUCTION}

The amygdala is a key brain region that in conjunction with the hippocampus and the prefrontal cortex supports fear memory formation and extinction (McGaugh, 2004; Milad and Quirk, 2002; Pape and Pare, 2010). While an extensive body of research has mostly focused on studying the role of excitatory synapses in fear memory formation, cumulative evidence suggests an important role of inhibitory synapses in modulating fear memory in the amygdala (Ehrlich et al, 2009; Lee et al, 2013). The interplay between excitatory principal cells and interneurons mediates local circuit activity (Maroun and Richter-Levin, 2002; Isaacson and Scanziani, 2011). Similarly to cortical structures, $10-15 \%$ of the neuronal population of the basolateral amygdala (BLA) is composed of a diverse group of GABAergic interneurons, which innervate distinct subcellular domains of projection neurons and are thought to shape fear memory (Ehrlich $e$ al, 2009; Li et al, 1996; Mascagni and McDonald, 2003;

*Correspondence: Professor G Richter-Levin, Sagol Department of Neurobiology, University of Haifa, Abba Khoushy Avenue 199, Haifa 31905, Israel, Tel: +972 48240962, Fax: +972 48288578, E-mail: galrichterlevin@gmail.com

Received 27 April 2016; revised 18 August 2016; accepted 22 August 2016; accepted article preview online 16 September 2016
Spampanato et al, 2011). Recent studies have begun to reveal a critical role of specific interneuron subpopulations in the BLA in different phases of fear memory formation and extinction (Bienvenu et al, 2012; Wolff et al, 2014). For instance, axo-axonic cells (AACs) are recruited in response to noxious stimuli and contribute also to the integration of hippocampal theta oscillation (Bienvenu et al, 2012), which is part of the memory consolidation process and is engaged during extinction learning (Lesting et al, 2011; Seidenbecher et al, 2003). During fear extinction, subsets of BLA projection neurons activated by fear conditioning are silenced by increased perisomatic inhibition originating from parvalbumin-positive interneurons (Trouche et al, 2013).

One possibility to control GABAergic inhibitory transmission of AACs emerges by manipulating neurofascin (NF) (Kriebel et al, 2011; Zitman et al, 2014). Neurofascin is a cell adhesion molecule belonging to the L1 subfamily of immunoglobulins (Kriebel et al, 2012). A neuronal isoform of NF is exclusively expressed at the innervation area of AACs, the axon initial segment (AIS). There, NF has been implicated in maintaining action potential initiation (Zonta et al, 2011) and in stabilizing inhibitory synapses by regulating the postsynaptic scaffold protein gephyrin (Burkarth et al, 2007; Kriebel et al, 2011). Knockdown of $\mathrm{NF}$ at the AIS decreased gephyrin clustering, which was 
associated with reduced expression of the $\mathrm{GABA}_{\mathrm{A}}$ receptor subunit $\beta 2 / 3$ at the postsynaptic site and GAD65 at the inhibitory presynapse (Zitman et al, 2014). Within the dentate gyrus (DG), such a modulation of GABAergic inhibitory transmission at the AIS via NF knockdown altered feedback inhibition measured by paired-pulse stimulation while feed-forward inhibition remained unchanged. This finding suggests a role of NF in specific aspects of local circuit activity. In addition, NF knockdown in the DG impaired avoidance learning in the two-way shuttle box, thus highlighting a functional role in stress coping (Zitman et al, 2014).

In the present study, we investigated how reduction of GABAergic synapses at the AIS of BLA projection neurons by NF knockdown affects cued fear conditioning and extinction as key paradigms recruiting the BLA. We assessed long-term potentiation (LTP) in the ventral subiculum (vSub)-BLA pathway, which represents the major connection between hippocampus and BLA as part of the fear conditioning and extinction network (Biedenkapp and Rudy, 2009; Hobin et al, 2006; Horovitz and Richter-Levin, 2015; Kim et al, 2013; Maren et al, 1995). Indeed, LTP was impaired after NF knockdown, confirming the functionality of the manipulation. While cued fear memory was intact, rats with NF knockdown displayed a selective deficit in fear extinction.

\section{MATERIALS AND METHODS}

Neurofascin knockdown was achieved via lentiviral miRNA expression vectors directed against all rat NF isoforms. Two target sequences were chosen (miNF1196 and miNF1707) and were cloned into vectors that provide the expression of miRNA sequences under the control of a short mouse CaMKII promoter and enhanced green fluorescent protein (EGFP) as a marker under the control of a short rat synapsin promoter (Dittgen et al, 2004). The miRNA-mediated reduction of NF expression was first validated in vitro in rat primary cortical neurons by comparing NF mRNA expression levels via quantitative PCR. We then proceeded to inject viral vectors bilaterally into the BLA (Figure 1c-e) of young adult (post-natal day (PND) 60) male SpragueDawley rats. After recovery from stereotactic injection for 2 weeks, either immunohistochemical and electrophysiological validation of the NF knockdown effect in vivo was performed in slice preparations, or rats were subjected to a battery of behavioral tests and subsequent in vivo electrophysiology (see experimental timeline Figure 1f), which was finalized by validation of injection and recording sites (Figure 1c-e; Supplementary Figures S6 and S9).

Immunohistochemical analysis (PND 74) comprised the identification of RNAi expressing neurons in the BLA via EGFP co-expression, followed by immunostainings for either Ankyrin G (AnkG) together with vGAT or gephyrin, or AnkG together with NF, which were always combined with blue fluorescent Nissl staining. Images were obtained with a laser-scanning microscope. Image analysis was performed in three dimensions on $Z$-stacks of quadruple fluorescence images. We quantified the lentiviral-induced knockdown of NF and the number of GABAergic synapses by pre- and postsynaptic markers (vGAT or gephyrin, respectively) on
AISs (using AnkG as a marker), and in addition, vGAT terminals on the somata of BLA projection neurons.

Slice electrophysiology (PND 81) was performed using standard procedures to assess cellular excitability and inhibitory synaptic transmission by measuring miniature inhibitory postsynaptic currents (mIPSCs) after NF knockdown in BLA projection neurons.

The behavioral analysis was comprised of an open-field (OFT; PND 74) and an elevated plus maze (EPM; PND 75) test (Ardi et al, 2016), followed by fear conditioning (PND 76-81) to an auditory cue and extinction by re-exposure to tones on 3 consecutive post-training days (adapted from Vouimba and Maroun, 2011).

In vivo electrophysiology (PND 82) was performed to test whether a high-frequency stimulation (HFS) of the vSub can induce plasticity in the BLA (adapted from Horovitz and Richter-Levin, 2015).

All methods are described in detail in the Supplementary Information.

\section{RESULTS}

\section{In Vitro Validation of Neurofascin Knockdown}

To specifically remove NF from projection neurons in the BLA, we designed lentiviral vectors that expressed miRNAs directed against NF or a control miRNA under control of a truncated CaMKII promoter together with EGFP controlled by a synapsin promoter (Figure 1a). To assess the efficacy of lentiviral constructs used for miRNA-mediated knockdown of NF, we quantitatively transduced primary cortical rat neurons with respective viral suspensions (Supplementary Figure S1). Expression of two different miRNAs specific for NF yielded markedly reduced levels of NF mRNA measured by quantitative real-time PCR when compared with control miRNA (CTR)-transduced cultures (experiments carried out in duplicate), indicating highly efficient suppression of NF mRNA expression (Figure 1b). The NF1707 miRNA (NF1707) construct appeared to be slightly more effective than the NF1196 miRNA (NF1196) construct.

\section{In Vivo Knockdown of Neurofascin Reduces GABAergic Input at the Axon Initial Segment}

To verify that lenitiviral knockdown of NF mRNA reduced its protein levels in vivo, quantitative immunohistochemistry was performed 2 weeks after viral injection (Supplementary Figure S2). We compared non-infected (EGFP-negative) with close-by infected (EGFP-positive) BLA projection neurons within the same reconstructed $Z$-stack after confocal image acquisition (Figure $2 \mathrm{a}-\mathrm{c}$ ). Using AnkG as a marker, we confirmed that the lengths of the AISs were similar in infected and non-infected neurons for all viral constructs $(\mathrm{CTR}, t(46)=-1.87, \quad P=0.068 ; \quad \mathrm{NF} 1196, \quad t(40)=-0.79$, $P=0.433$; NF1707, $t(38)=-0.343, P=0.735$; Figure $2 \mathrm{~d})$, indicating no major structural impairment of the AIS. Knockdown with NF1707 led to a significant reduction in NF staining at the AIS $(t(19)=-3.493, P=0.003)$, while the CTR was ineffective $(t(24)=-0.577, P=0.569)$. No reduction of NF signal was observed with NF1196 $(t(20)=-0.323$, $P=0.750$ ), although this construct reduced mRNA levels, suggesting that the reduction in protein expression was too 
a

5' LTR - $\Psi$ RRE CaMKII -

$-5^{\prime}$ LTR $-\Psi-$ RRE $-\underbrace{\text { CaMKII }}_{\substack{\text { att81 } \\ \text { miNf1196 }}}$
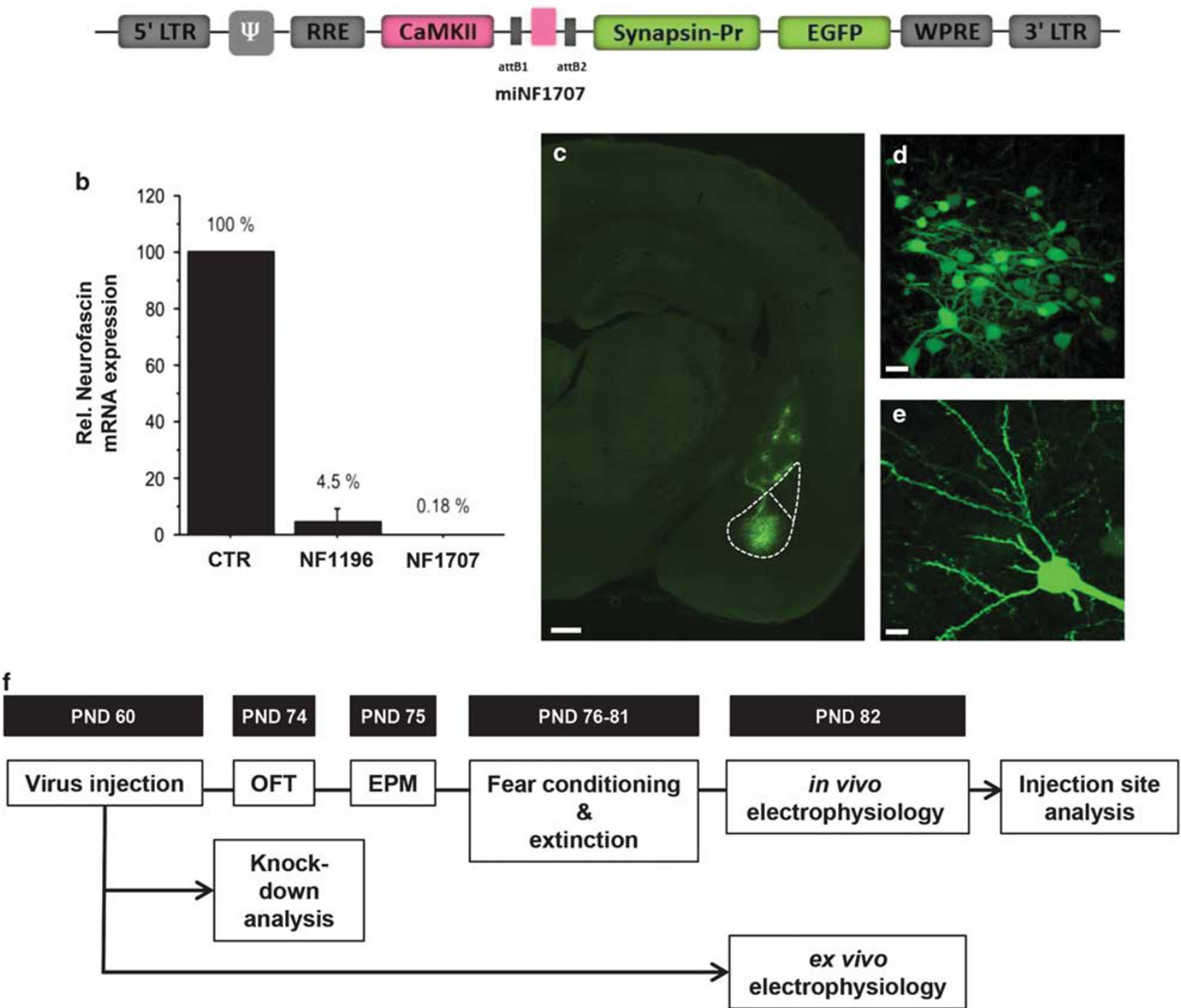

Figure I Knockdown of neurofascin via lentiviral-mediated RNA interference. (a) Structure of viral vectors (pLenti04C_miRNA/SEW) used for RNAi induced knockdown in vitro and in vivo. Respective miRNA-oligos are expressed under control of a shortened mouse CaMKIl promoter, and EGFP under the control of a rat synapsin promoter to visualize transduced neurons. $\Psi$, HIV-I psi packaging signal; RRE, Rev response element; WPRE, Woodchuck hepatitis virus post-transcriptional regulatory element. (b) Evaluation of knockdown efficacy in vitro. Primary rat cortical neurons were analyzed by TaqMan Real-Time PCR for the expression of neurofascin mRNA after quantitative transduction with lentiviral suspensions causing expression of CTR (control miRNA), or neurofascin-specific NFII96 and NFI707 (NFII96 miRNA and NFI707 miRNA). Expression of both NFI 196 and NFI707 resulted in a substantial reduction of neurofascin mRNA compared with control $(n=2$, data are shown as means \pm SEM). (c) Representative image of EGFP expression in the BLA after injection of lentiviral vector. Scale bar: $500 \mu \mathrm{m}$. The BLA is outlined. (d) Magnification of NFI707-infected cells within the BA. Scale bar: $20 \mu \mathrm{m}$. (e) Magnification of a NFI707-infected cell in the BA with dendrites and spines. Scale bar: $10 \mu \mathrm{m}$. (f) Scheme of experimental timeline, PND, postnatal day.

weak to detect by immunostaining (Figure 2e). In a next step, we assessed the impact of NF knockdown on the density of presynaptic vesicular GABA transporter clusters used as marker for GABAergic terminals (Chaudhry et al, 1998). We found that loss of NF with NF1707 was accompanied by a highly significant reduction of vGAT-positive terminals at the AIS (by $38 \%(t(19)=7.011, P<0.0001$ ), which did not occur with CTR expression $(t(22)=0.729, \quad P=0.474$; Figure 2f). There was also a smaller, yet significant decrease of vGAT terminals at the AIS after expression of NF1196 by 
a

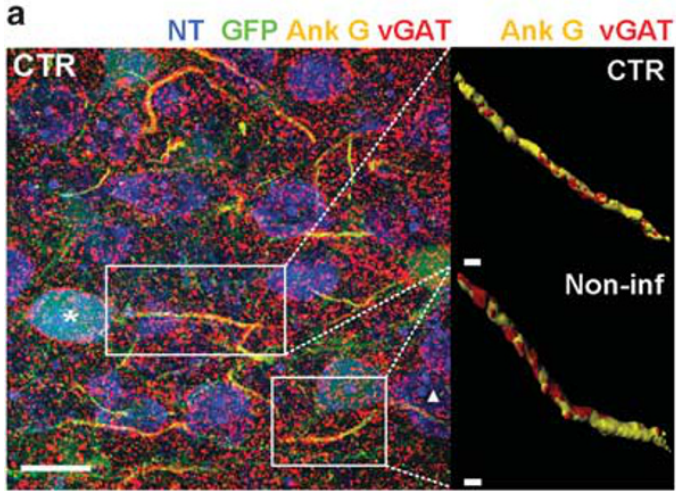

b

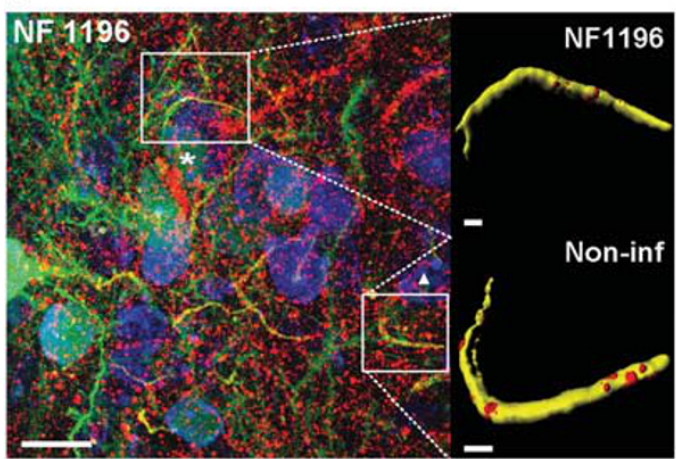

C

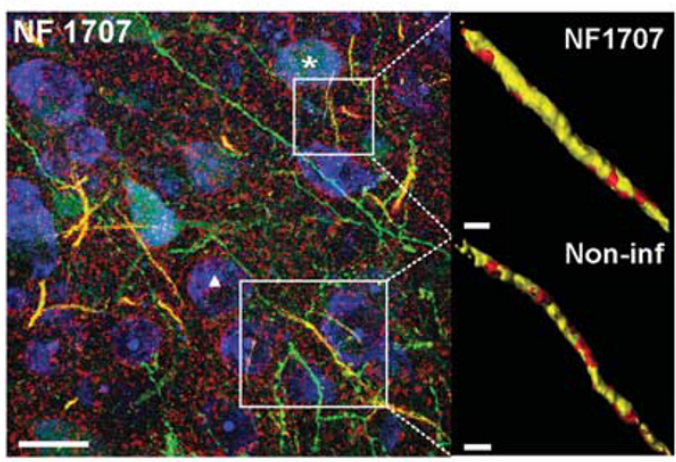

g

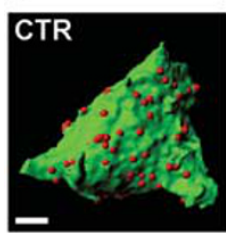

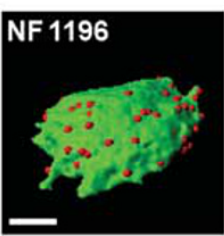

d
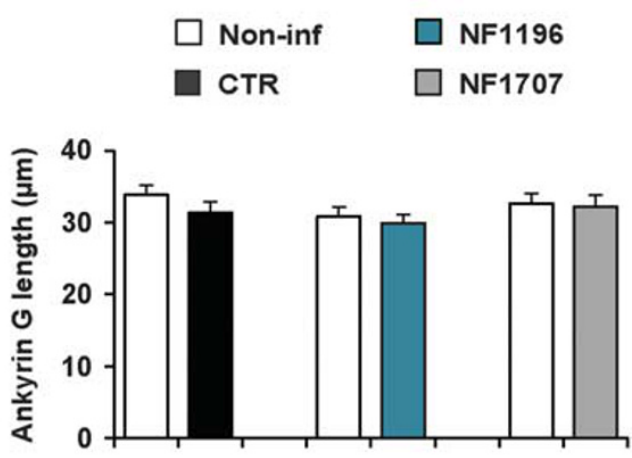

e

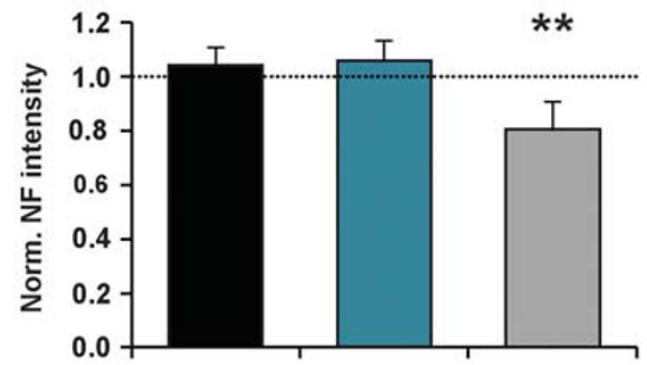

f
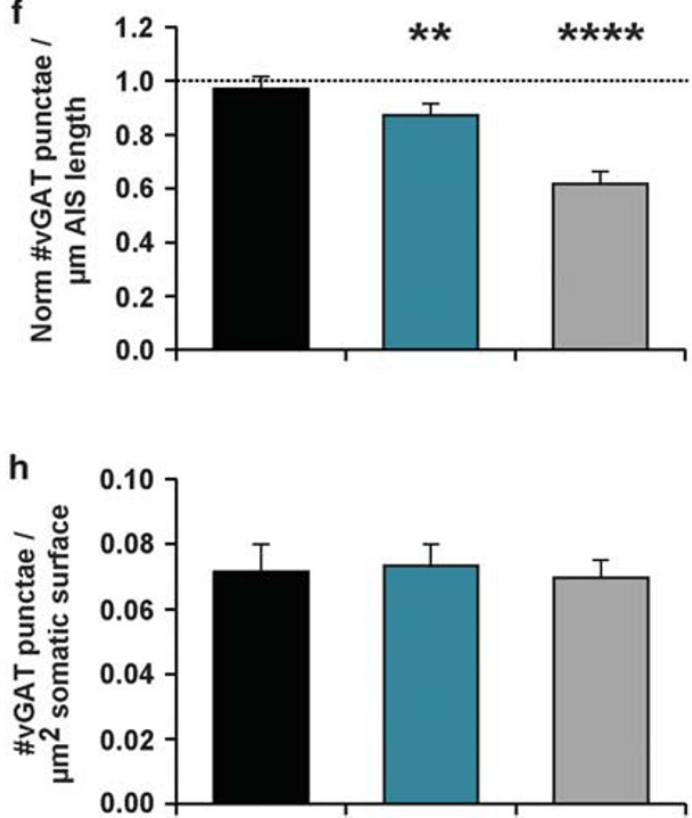

Figure 2 Knockdown of neurofascin specifically decreases GABAergic terminals at the axon initial segment. (a-c) Maximum intensity projections of confocal Z-stacks of the BLA showing CTR (a)-, NFI 196 (b)-, and NFI 707 (c)-infected cells (asterisk) and non-infected cells (arrowhead). Infected cells express EGFP (green), all somata were visualized with Neurotrace (blue), AISs were visualized with Ankyrin G (yellow), and GABAergic terminals were visualized with the presynaptic marker vGAT (red). Scale bars: $20 \mu \mathrm{m}$. Insets show 3D reconstructions of extracted AISs of infected- and non-infected cells with vGAT terminals. Scale bars: $2 \mu \mathrm{m}(\mathrm{a}, \mathrm{b}), 5 \mu \mathrm{m}$ (c). (d) Comparison of AIS length did not differ in any of the analyzed BLA cells (non-infected vs CTR-infected, $n=46$ each; noninfected vs NFI 196-infected $n=40$ each; non-infected vs NFI 707-infected, $n=38$ each). (e) Neurofascin levels assessed as normalized neurofascin intensity (Inf/Non-inf) were reduced by NFI 707 ( $n=19 / 19)$, but not in CTR $(n=24 / 24)$ or NFI $96(n=20 / 20)$. (f) The density of vGAT-positive terminals at the AIS assessed as normalized density (Inf/Non-Inf) was reduced by neurofascin knockdown (NFI707, $n=19 / 19 ;$;FI I96, $n=20 / 20$ ), but not in CTR ( $n=22 / 22$ ). (g) 3D reconstructions of EGFP-labeled somata (green) with somatic VGAT terminals (red) for CTR-, NFI I 96-, and NFI 707-infected cells. Scale bars: 5 um. (h) The density of somatic vGAT punctae did not differ between constructs (CTR $n=15, \mathrm{NFI} 196 n=15, \mathrm{NFI}$ 707 $n=15)$. Data are shown as means \pm SEM. Asterisks indicate significant differences (** $P<0.01$, ***** $P<0.000$ I). 
$13 \%(t(20)=3.179, P=0.005$; Figure $2 f)$, confirming the partial efficacy of this construct. The overall coverage and distribution of vGAT-positive terminals at the AIS was in good agreement with previously published data (cf. Supplementary Figure S3; Veres et al, 2014). The relative distribution was not altered by miRNA expression, indicating that vGAT terminals were uniformly reduced along the AIS (Supplementary Figure S3). Importantly, GABAergic terminals at neuronal somata were not altered and also did not differ between the viral constructs used $(\mathrm{F}(2,44)=0.136$, $P=0.873$ ) (Figure $2 \mathrm{~g}$ and $\mathrm{h}$ ), indicating that perisomatic GABAergic synapses were unaffected. In addition, we used gephyrin as postsynaptic marker of GABAergic synapses (Craig et al, 1996) to confirm loss of synapses. Again, AnkG staining confirmed that the lengths of the AISs were unaltered with viral transduction (CTR vs non-inf., $t(8)=0.326, P=0.754$; NF1707 vs non-inf., $t(12)=-0.223$, $P=0.828$; Supplementary Figure S4c), indicating no major structural impairment of the AIS. Loss of NF with expression of NF1707 led to a significant reduction of gephyrin clusters along the length of the AIS by $30 \%$ ( $v s$ non-inf., $t(12)=$ -0.793 ; $P<0.001$; Supplementary Figure S4b, $d$ and e), which did not occur with CTR expression ( $v s$ non-inf. $t(8)=-6.595 ; P=0.454$; Supplementary Figure S4a, $d$ and e).

Together, our data show that two independent NF-specific miRNAs impaired GABAergic innervation at the AIS, but not at the soma, suggesting no off-target effects. However, in vivo expression of NF1707 reduced NF protein levels in BLA projection neurons more effectively. Thus, we focused our main analysis on physiological and behavioral effects on NF1707, while results from NF1196 are given in the Supplementary Information, and provide an important comparison.

\section{Neurofascin Knockdown in BLA Alters mIPSCs}

To evaluate the impact of NF knockdown on synaptic transmission, mIPSCs (Figure 3a) were measured in brain slices from lentivirus-infected rats. Comparison of EGFPpositive, NF1707 $(n=28)$ and CTR $(n=25)$-infected neurons revealed that knockdown of NF resulted in a significant leftward shift in the amplitude distribution (CTR vs NF1707, for amplitude, $P<0.001, \mathrm{~K}-\mathrm{S}$ test; Figure $3 \mathrm{~b}$ ) and a reduction in the average mIPSC amplitude ( $t$-test, $t(51)=2.163$, $P<0.01$; Figure $3 \mathrm{~d}$ ). Conversely, the distribution of interevent intervals was unaltered (CTR vs NF1707, for interevent interval, $P>0.05, \mathrm{~K}-\mathrm{S}$ test; Figure $3 \mathrm{c}$ ) and the average mIPSC frequency were unaltered $(t(51)=0.329, P=0.743$; Figure $3 \mathrm{e})$. Together, this suggests a functional postsynaptic reduction of GABAergic synaptic transmission by NF knockdown in BLA projection neurons.

\section{Neurofascin Knockdown Reduces Cellular Excitability in BLA}

Next, we measured cellular excitability from EGFP-expressing neurons infected with CTR $(n=14)$ or NF1707 $(n=15)$ in BLA projection neurons. We injected a series of depolarizing current steps to assess the number of action potentials (Figure $4 \mathrm{a}-\mathrm{c})$. Repeated-measure ANOVA revealed that projection neurons fired significantly less after NF knockdown (group $\times$ current steps intensity interaction: $F(1.973,53.279)=19.648$,
$P<0.001$, Greenhouse-Geisser correction) (Figure 4d). In addition, NF knockdown led to a significant hyperpolarization of the resting membrane potential $(t(27)=4.192, P<0.001$; Supplementary Figure S5a), while the action potential threshold was significantly depolarized $(t(27)=3.820, \quad P<0.01$; Supplementary Figure S5b). As a result, the threshold current was significantly increased in NF1707-infected neurons $(t(27)=4.487, P<0.001$; Supplementary Figure S5c), while the input resistance was unaltered $(t(27)=0.036, P=0.971$; Supplementary Figure S5d). Moreover, the action potential half-width was significantly increased after NF knockdown $(t(27)=2.735, P<0.05$; Supplementary Figure S5f), while the amplitude was not altered $(t(27)=1.591, \quad P=0.123$; Supplementary Figure S5e). Together, these experiments indicate that NF knockdown reduces firing patterns and compromises cellular excitability of BLA projection neurons.

\section{Neurofascin Knockdown Impairs BLA Synaptic Plasticity}

To address the functional physiological consequences of NF knockdown, we turned to in vivo recordings, and examined properties of vSub-evoked field potentials in the BLA in animals with confirmed viral expression and recording sites (Supplementary Figure S6). Comparing NF1707 $(n=10)$ and CTR $(n=8)$ groups, no significant effect on basal excitability, as assessed by input-output curves, was found despite a trend toward increased excitability (Supplementary Figure S7; $\mathrm{F}(1,16)=2.280, \quad P=0.151)$. Repeated-measure ANOVA showed no group $\times$ intensity interaction ( $F$ $(2.080,33.27)=1.984, P=0.152$, Greenhouse-Geisser correction). Upon application of HFS, vSub-evoked field potentials in the BLA were effectively potentiated in the control group, but not in the NF knockdown group as revealed by mixed model repeated-measure ANOVA (group effect: (F $(1,16)=16.27, P=0.001$; and group $\times$ post-HFS time point interaction: $F(2.071,33.136)=9.231, P=0.001$, GreenhouseGeisser correction; Figure 4f). The comparison of average potentiation across time points after induction also revealed a highly significant difference between the two groups (Figure 4g, $t(16)=4.03, P=0.001)$. NF knockdown with the less effective NF1196 vector also reduced vSub-BLA LTP (Supplementary Figure S8b and c) without altering the input-output curve (Supplementary Figure S8a). Together, this demonstrates a specific impairment of BLA synaptic plasticity to vSub inputs after NF knockdown.

\section{Neurofascin Knockdown Does Not Alter Acquisition and Expression of Cued Fear}

To assess the impact on fear behavior, we first examined whether NF knockdown affects acquisition of conditioned fear. Animals expressing NF1707 or CTR ( $n=13$ per group; see Supplementary Figure S9 for verification of virus expression) both displayed low pre-conditioning basal freezing levels in context A $(t(24)=0.152, P=0.881$; Figure 5a). During the training phase, freezing increased over the three conditioned stimulus (CS)-US pairings in both groups (Figure 5b) as assessed by repeated-measure ANOVA (effect of tones: $\mathrm{F}(2,48)=214.9, P=0.001$; no group effect: $\mathrm{F}(1,24)=0.146$, $P=0.706$; no group $\times$ tone interaction: $\mathrm{F}(2,48)=1.99, P=0.14)$ and remained at comparable levels in $2 \mathrm{~min}$ post training 
a
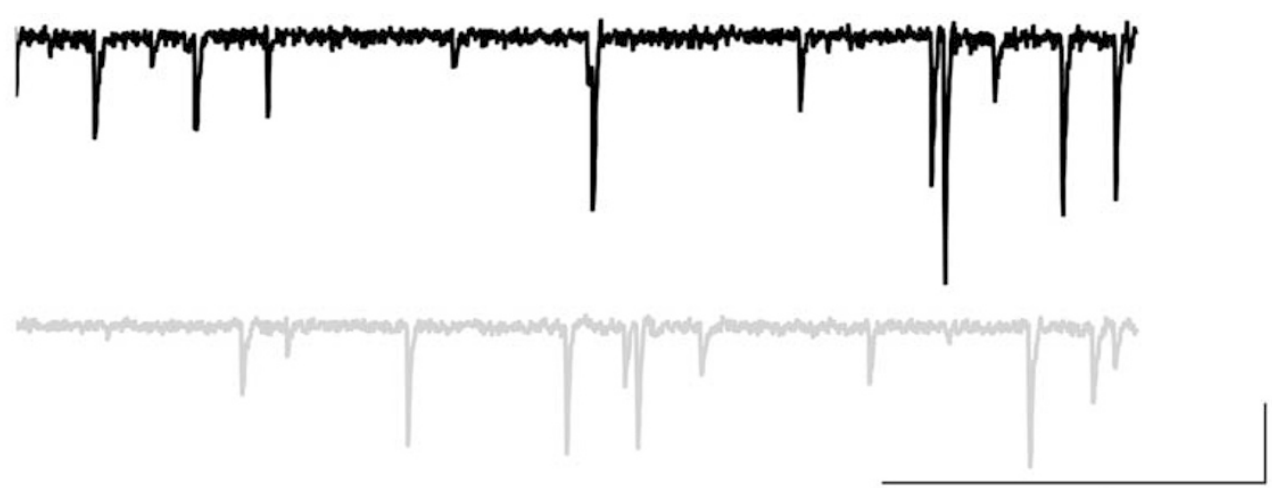

$20 \mathrm{pA}$

$1 \mathrm{~s}$
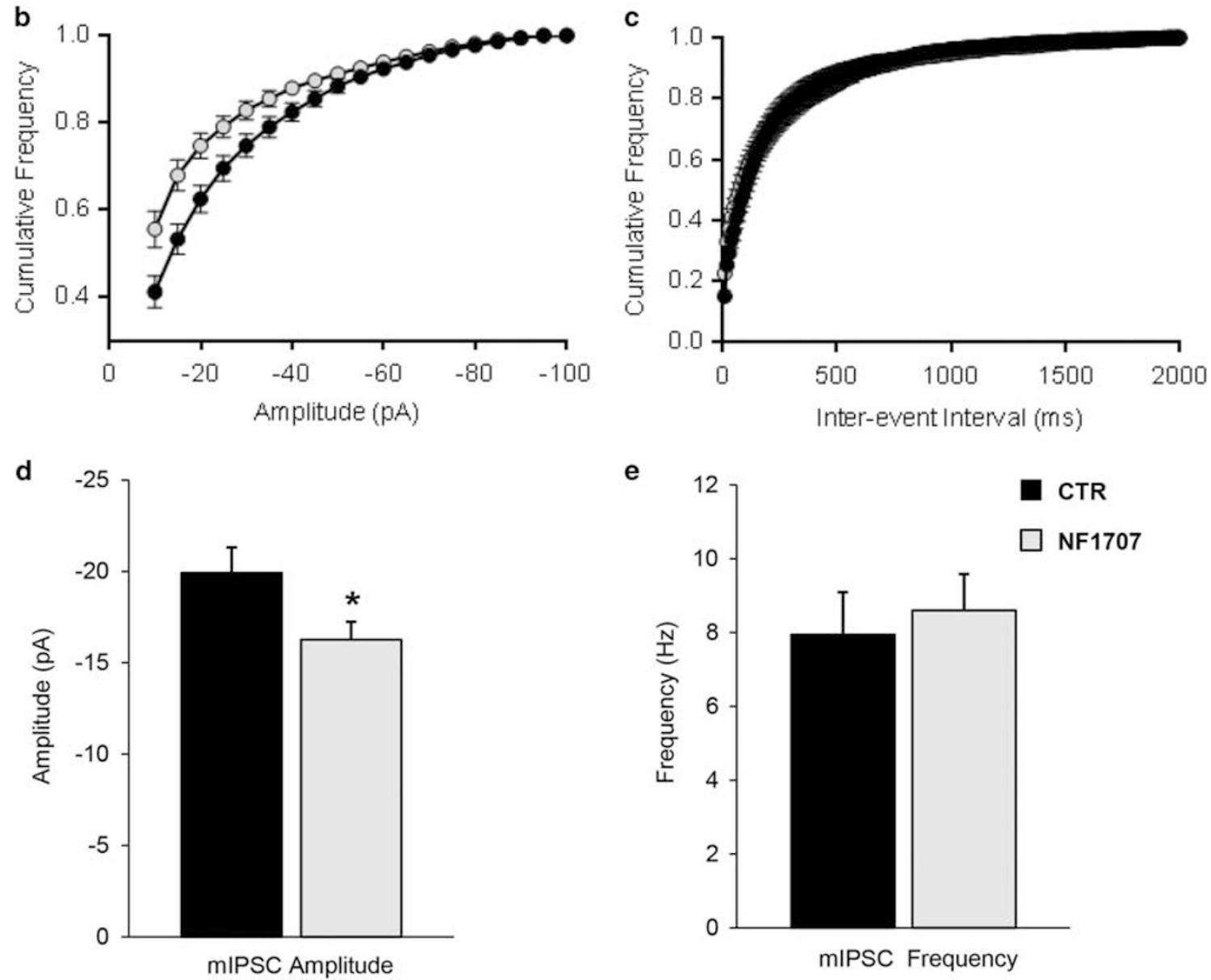

Figure $3 \mathrm{mIPSC}$ amplitude but not frequency is altered by neurofascin knockdown in the basolateral amygdale. (a) Representative traces of whole-cell voltage-clamp recordings of mIPSCs obtained from BLA projection neurons infected with CTR (black) or NFI 707 (gray) vector. (b) A significant leftward shift is observed for the cumulative frequency of amplitudes recorded from NFI707 $(n=28)$ - versus the CTR ( $n=25$ )-infected neurons. (c) Inter-event interval was not different between the two groups. (d) The average amplitude of mIPSCs was significantly reduced in the NFI707 group, while (e) the mIPSC average frequency remained unaltered in NFI707 compared with CTR-vector-infected neurons. (d, e) Data are shown as means \pm SEM. Asterisk indicates significant difference $(* P<0.05)$.

Figure 4 Cellular excitability and long-term potentiation is diminished by neurofascin knockdown in the basolateral amygdala. (a, b) Representative recording traces of action potential firing from CTR (black)- and NFI707 (gray)-infected neurons to the current injection protocol indicated in (c). (d) Significant reduction in the frequency of AP firing in NFI707 $(n=15)$ compared with CTR-vector-infected $(n=14)$ neurons. (e) Representative evoked field potential responses recorded from the BLA in CTR (black) and NFI 707 rats (gray) before and after HFS. ( $)$ HFS to the vSub pathway induced a significant potentiation of BLA field potentials in CTR rats, which was reduced in NFI 707 rats. (g) The average \% change in peak height was significantly higher in the CTR compared with the NFI707 group. Data are shown as means \pm SEM. Asterisks indicate significant differences $(* * * P<0.00 \mathrm{I})$. 
$(t(24)=0.171, P=0.866$; Figure $5 \mathrm{c})$. Furthermore, animals in both groups retrieved the acquired fear and showed similar levels of freezing when tested the next day (Figure 5d, first five CS presentations at the beginning of extinction training $t(24)=-1.028, P=0.321)$. Also, the less effective NF1196 did not alter baseline freezing, fear acquisition or fear expression (Supplementary Figure S10a-d). Together, this shows that NF knockdown in the BLA did not alter fear learning and memory. a
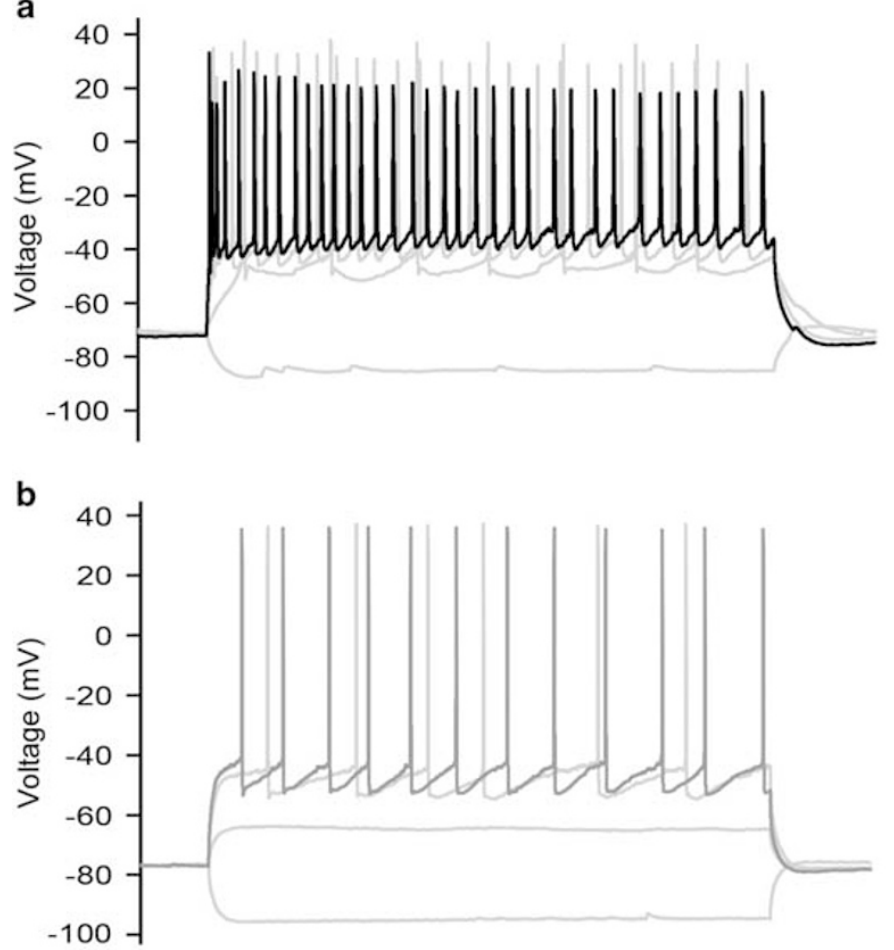

C

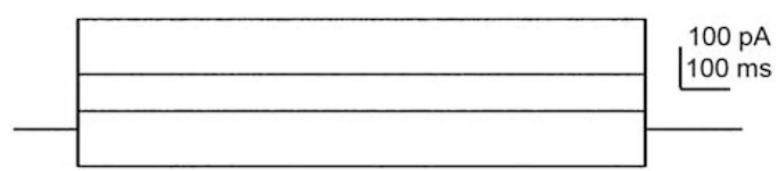

e
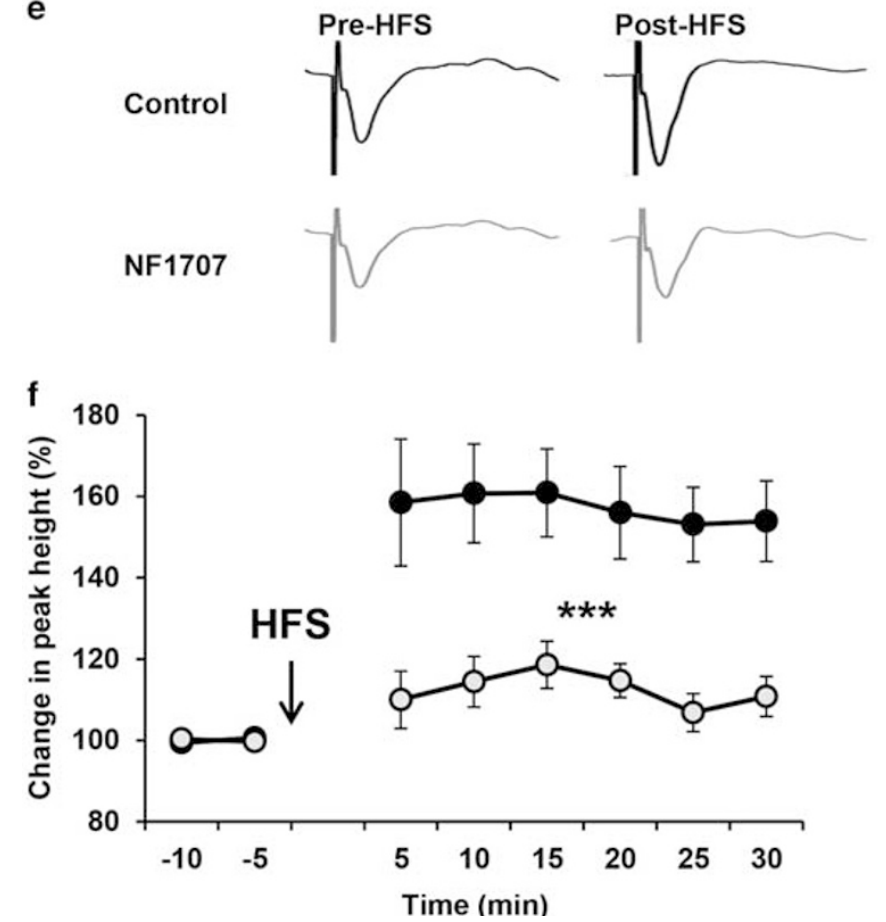

d

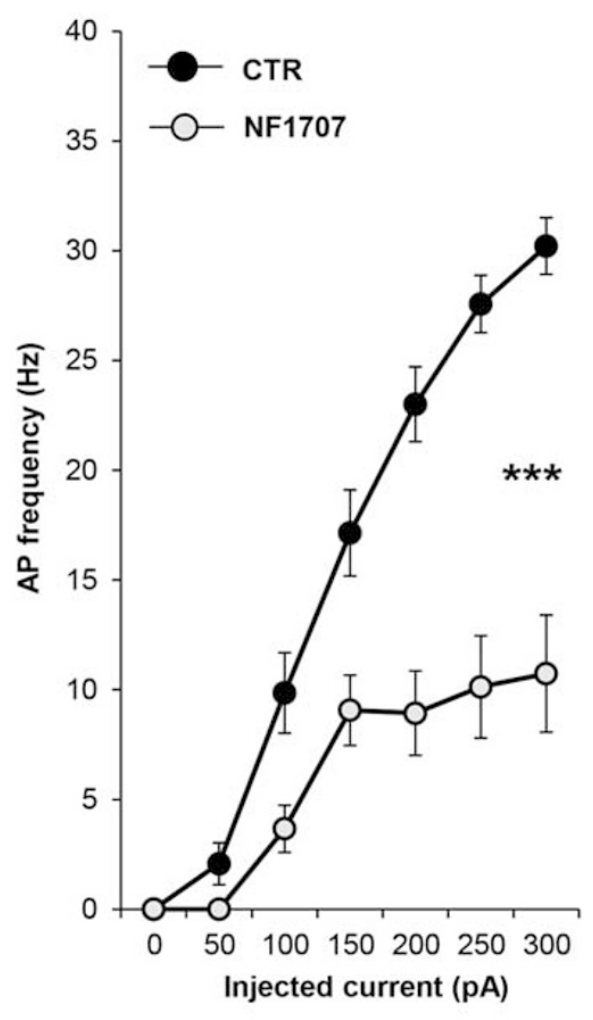

$\overline{5 \mathrm{~ms}}_{\substack{\mathrm{E} \\ \text { N }}}$

g

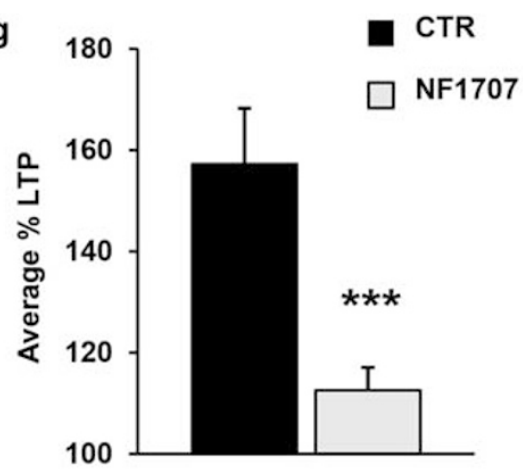




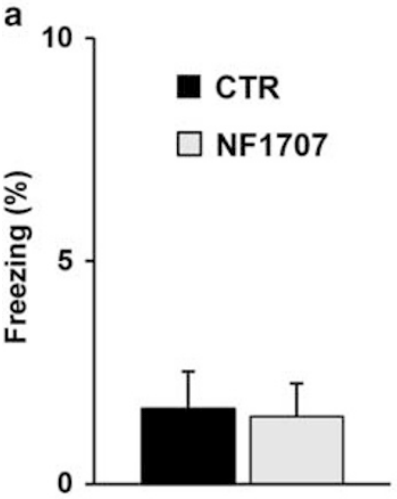

b

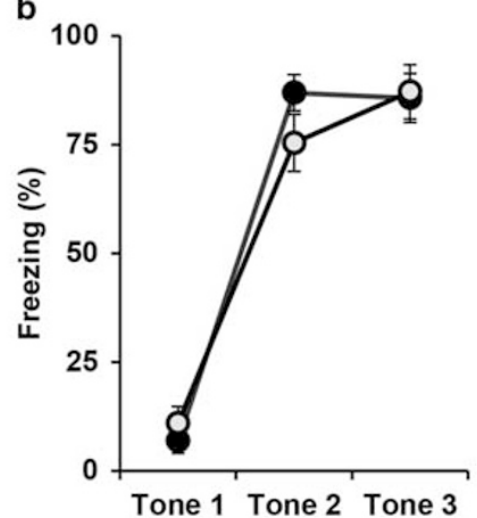

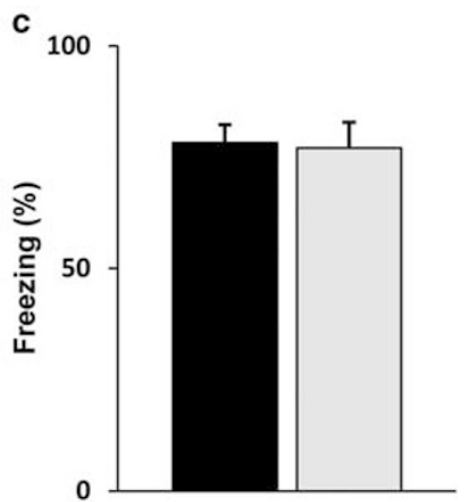

d

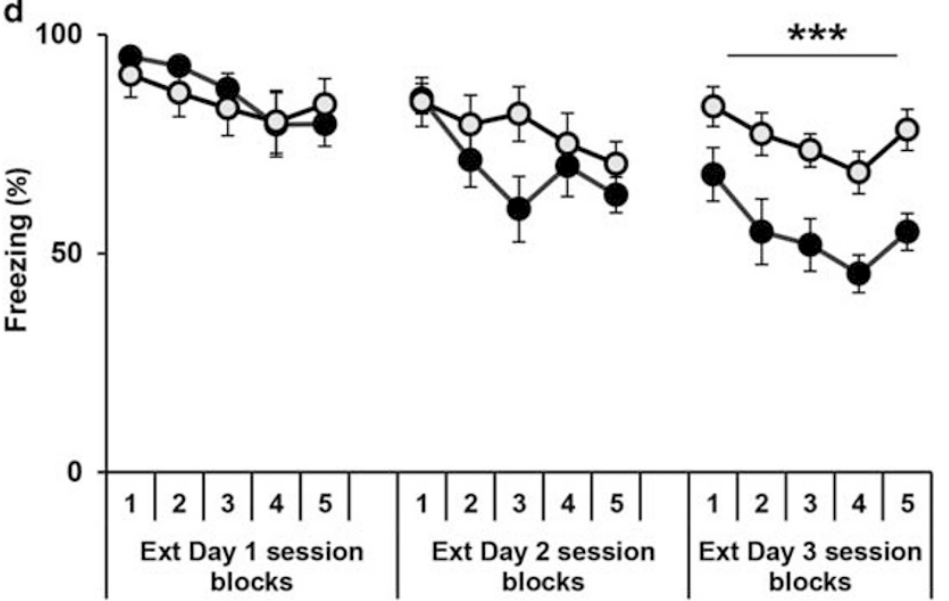

Figure 5 Extinction, but not cued fear conditioning is impaired by neurofascin knockdown in the basolateral amygdala. (a) On the conditioning day, CTR and NFI707 groups showed no significant difference in basal freezing levels during 2 min of pretraining exploration. (b) Acquisition of fear to the auditory conditioned stimulus (CS) paired with shock, measured as \% freezing to the CS, did not differ between animals with bilateral expression of CTR ( $n=13$ ) and NFI707 vectors $(n=13)$. (c) The post-shock freezing levels were comparable in CTR and NFI707 groups. (d) Twenty-four hours after conditioning, both groups underwent 3 days of extinction. Freezing levels to the CS were still higher in the NFI 707 vs the CTR group on day 3, but not during the two previous sessions indicating impairment in fear extinction memory. (e) Freezing difference between the initial (ext day I) and late phase (ext day 3) freezing (\%) response showing significantly low freezing difference in NFI 707 knockdown group. Data are shown as means \pm SEM. Asterisks indicate statistical significance between groups (*** $P<0.00 \mathrm{I})$.

\section{Neurofascin Knockdown Impairs Extinction of Conditioned Fear}

Twenty-four hours after fear conditioning, we administered a 3-day extinction training protocol in context $\mathrm{B}$, where animals were exposed to five blocks of two CS each day (Figure 5d). While no differences in baseline freezing at the start of extinction training were apparent (Supplementary Figure S11), a mixed model repeated-measure ANOVA revealed significant differences in freezing over extinction blocks between the NF1707 and CTR groups $(\mathrm{F}(10.63,255.16)=12.13, \quad P<0.001)$ with a significant block $\times$ group interaction $\quad(\mathrm{F}(10.63,255.16)=3.44, \quad P<0.001$, Huynh-Feldt correction), but no general group effect $(\mathrm{F}(1,24)=3.12, P=0.090)$. Post hoc comparisons revealed that a significant group difference emerged on day 2 of extinction. The within-session analysis revealed a significant difference between the groups only on day 3 of extinction (main group effect: $\mathrm{F}(1,24)=19.145, \quad P=0.001 ; \quad$ no block $\times$ group interaction: $\mathrm{F}(4,96)=0.276, P=0893$, Huynh-Feldt correction), but not on day 1 (group: $\mathrm{F}(1,24)=0.099, P=0.755$; block $\times$ group interaction: $\mathrm{F}(3.62,86.81)=0.786, P=0.526$, Huynh-Feldt correction) or day 2 (group: $\mathrm{F}(1,24)=1.489, \quad P=0.234$; block $\times$ group interaction: $\mathrm{F}(3.64,87.36)=2.086, P=0.096$, Huynh-Feldt correction). The between-session analysis (comparing the last block with the first block of the session on the following day) did not reveal any group differences from extinction day 1 to day 2 (group: $\mathrm{F}(1,24)=0.094$, $P=0.761$; block $\times$ group interaction: $\mathrm{F}(1,24)=0.483, P=0.494$, Huynh-Feldt correction) or from day 2 to day 3 (group: $\mathrm{F}(1,24)=3.689, \quad P=0.067 ; \quad$ block $\times$ group interaction: $\mathrm{F}(1,24)=1.126, P=0.299$, Huynh-Feldt correction). However, further analysis of average freezing in the initial phase (first five CSs on day 1) vs the late phase (last five CSs on day 3) of extinction confirmed that NF knockdown impaired cued fear extinction (first five CSs: $t(24)=-1.028, P=0.321$; last five CSs: $t(24)=5.02, P<0.001)$. Accordingly, the overall reduction of difference in freezing percentage (first five CSs - last five CSs) was significantly reduced in the NF1707 vs the CTR group (Figure 5e; $t(24)=4.31, P<0.001)$. With NF1196, a similar trend toward 
decreased extinction was observed (Supplementary Figure S10d and e). In summary, these data suggest that knockdown of NF impaired long-term memory of extinction for cuedconditioned fear.

\section{Neurofascin Knockdown Does Not Affect Locomotor Activity and Anxiety-like Behavior}

Finally, to rule out the possibility that the behavioral analysis of fear and extinction learning was confounded by changes in activity or anxiety-related behavior, we analyzed the rats' behavior in the OFT and EPM test before fear conditioning. Neurofascin knockdown by NF1707 did not affect activity and anxiety-like behavior, as assessed by total distance moved (Supplementary Figure S12a; $t(24)=0.99, P>0.05$ ) or by percentage of distance moved in the center of the openfield arena (Supplementary Figure S12b; $t(24)=0.57$, $P>0.05)$. Likewise, neither the total distance moved in the EPM arena (Supplementary Figure S12c; $t(24)=1.61$, $P>0.05)$ nor the percentage of distance or the duration moved in the open arms showed any significant differences between groups (Supplementary Figure S12d and e; $P>0.05$ ). Similarly, NF1196 had no significant effect on anxiety-like behavior in all of these behavioral assays (Supplementary Figure S13). Together, these data strengthen the notion that knockdown of NF specifically impaired fear extinction memory without affecting anxiety-related behavior.

\section{DISCUSSION}

In the present study we investigated the role of AIS GABAergic synapses in BLA projection neurons in fear behavior by knocking down the neural cell adhesion molecule NF, which is associated with the stabilization of these synapses (Burkarth et al, 2007; Kriebel et al, 2011). In the hippocampus, NF controls the assembly of GABAergic synapses at the AIS (Kriebel et al, 2011). A recent study from our lab demonstrated that loss of these synapses in dorsal DG impacts local network activity and behavior, selectively increasing feedback inhibition and impairing hippocampaldependent aversive learning in the two-way shuttle paradigm (Zitman et al, 2014). Here we demonstrate that a specific knockdown of NF in the BLA (1) exclusively reduced a pool of GABAergic synapses innervating the AIS of BLA projection neurons, resulting functionally in reduced GABAergic transmission and changes in excitability; (2) induced a deficit in LTP in the vSub-BLA pathway in vivo; and (3) impaired extinction while acquisition and expression of cued fear memory was intact, and anxiety-like behavior were unaltered. Together, this suggests that the manipulation of NF, resulting in a selective removal of AIS inhibitory synapses at BLA projection neurons, leads to a specific impairment of fear memory extinction.

Both NF-specific miRNAs were highly efficient in reducing $\mathrm{NF}$ mRNA in primary culture. In vivo reduction of $\mathrm{NF}$ protein expression was moderate for NF1707 and undetectable for NF1196. The discrepancy may be explained by the limited sensitivity of immunohistological analyses, or alternatively, by the stability of the NF protein. In the cerebellum, reduction of NF protein levels was observed in a tamoxifeninduced conditional mouse model only after 6 weeks while a synaptic phenotype was observed only after 16 weeks (Zonta et al, 2011). Effects of NF1196 were observed by in vivo electrophysiology performed more than 3 weeks after virus injection, while we were unable to detect effects on fear memory extinction after 2 weeks. Therefore, it is possible that a fully effective manipulation of NF levels with the NF1196 constructs is time-dependent. However, we observed synaptic alterations 2 weeks after lentiviral infection with NF1707 without overt alterations of the AIS based on AnkG analysis. The synaptic effects are well in line with earlier hippocampal studies (Kriebel et al, 2011; Zitman et al, 2014) showing a relatively rapid reduction of GABAergic synapses at the AIS, while perisomatic synapses were unaffected. Both NF knockdown constructs reduced GABAergic terminals at the AIS, but to a different extend, which is in line with their differential effect on extinction learning. Together, these findings indicate that our manipulation was specific and rules out off-target effects.

Because the AIS is exclusively targeted by AACs (Howard et al, 2005; Woodruff et al, 2010), NF knockdown likely perturbed the signaling of this specific set of interneurons onto BLA projection neurons. This notion was further supported by the observed reduction of mIPSC amplitude after NF knockdown. Usually, a change in the amplitude of mIPSCs reflects alterations in postsynaptic $\mathrm{GABA}_{\mathrm{A}}$ receptors while a change in the frequency of mIPSCs implicates an altered probability of transmitter release and/or changes in the number of active synapses (De Koninck and Mody, 1994; Otis et al, 1994). The reduction in average amplitude after NF knockdown may result from a decreased frequency of larger events, probably due to a reduction of number of postsynaptic $\mathrm{GABA}_{\mathrm{A}}$ receptors (Otis et al, 1994). However, the lack of observed alterations in the frequency could be explained by the fact that relative to the overall GABAergic input, only a very small number of synapses were completely removed at the AIS, while smaller events remained. Nevertheless, our results provide direct evidence that NF knockdown impairs GABAergic transmission within the BLA by reducing functional $\mathrm{GABA}_{\mathrm{A}}$ receptors at the postsynaptic site.

Moreover, due to its restriction to the AIS, knockdown of $\mathrm{NF}$ and the associated reduction in inhibitory inputs may also affect the properties of evoked action potentials. After NF knockdown, the firing rate was significantly reduced and the action potential half-width was increased, while the input resistance was unaltered. The reduced firing rate of neurons could be explained by their hyperpolarized resting membrane potential and a depolarized threshold potential, in accordance with Zonta et al (2011). Indeed, it has been demonstrated that AACs can control action potential generation at the AIS of projection neurons via their GABAergic inputs, either through changing the action potential threshold, or by shifting the site of origin (Douglas and Martin, 1990; Somogyi et al, 1982; Wefelmeyer et al, 2015; Veres et al, 2014). Accordingly, similar differences in action potential properties as seen after NF knockdown arise by shifting the action potential initiation site to the first node of Ranvier (Clark et al, 2005). Moreover, the reduced firing rate of projection neurons could also contribute to the observed LTP impairment (Cohen et al, 1999). 
To investigate BLA-LTP in vivo, we chose the vSub-BLA pathway, which represents the major connection between hippocampus and BLA (Horovitz and Richter-Levin, 2015; Kim et al, 2013; Maren et al, 1995) and is known to be part of the fear conditioning and extinction circuitry (Biedenkapp and Rudy, 2009; Hobin et al, 2006). While impaired LTP in the vSub-BLA pathway after NF knockdown in the BLA may result from reduced neural excitability, its relation to reduced GABAergic inhibition by NF knockdown appears counterintuitive on first sight. However, it may be related to the fact that previous studies suggested rather excitatory net effects of AACs onto BLA projection neurons (Woodruff et al, 2010). Monosynaptic vSub-BLA projections exist (Mello et al, 1992), and can recruit projection neurons, but also local interneurons via feed-forward circuits, but the role of AACs remains unclear (Bazelot et al, 2015; Hübner et al, 2014). However, vSub afferents represent only a minor part of BLA afferents (for example, Canteras et al, 1992) and AACs are also reciprocally connected to projections neurons, suggesting they could also be part of local feedback circuits (Veres et al, 2014). Accordingly, vSub-BLA plasticity may be also shaped by intra-amygdalar local inhibitory networks. Cumulative evidence suggests that different BLA afferents target specific subpopulations of interneurons (Leitermann et al, 2016; McDonald et al, 2011; Unal et al, 2014), thus enabling input-specific modulation of BLA local networks, which in turn may affect induction of LTP in this structure. Notably, the input-output curve revealed a trend for increased BLA excitability in the NF1707 knockdown group, which could reflect local network changes that may affect further potentiation. As all animals received fear conditioning and extinction training before the BLA-LTP recordings in vivo, baseline excitability and plasticity could have been affected by this experience in interaction with the NF knockdown effects. Nevertheless, this was not observed for the NF1196 construct, while LTP was similarly reduced. This strongly suggests that impaired LTP was not a result of a priori increased excitability.

Within the extinction network, activity and plasticity of the BLA is considered critical for expression of fear, as well as the initial acquisition of extinction memory (Herry et al, 2008; Quirk and Mueller, 2008). Knockdown of NF in the BLA did not affect initial cued fear memory acquisition and consolidation, nor did it affect basal levels of anxiety-related behaviors, suggesting a specific extinction deficit (Dalton et al, 2008). This idea is in line with the role of NF in GABAergic transmission. Several studies converge on showing that extinction training globally increases GABAergic markers including gephyrin and $\mathrm{GABA}_{\mathrm{A}}$ receptor subunits in the BLA (Chhatwal et al, 2005; Heldt and Ressler, 2007; Lin et al, 2009), and interfering with $\mathrm{GABA}_{\mathrm{A}}$ receptor insertion by a TAT-conjugated peptide within BLA synapses impaired fear extinction (Lin et al, 2009). However, the role of specific interneuron subtype inputs remains less well understood. To the best of our knowledge, only one study addressed the role of such specific changes, demonstrating remodeling of perisomatic synapses in contextual fear extinction (Trouche et al, 2013). Knockdown of NF reduced GABAergic markers at the AIS, supporting the view that increased GABAergic transmission at this compartment is also required for extinction learning.
Disrupting the structural integrity of the AIS, for example, in Angelman syndrome due to increased expression of AnkG and the $\mathrm{Na} / \mathrm{K}$ ATPase subunit $\alpha 1$, can indeed lead to impaired contextual fear memory (Kaphzan et al, 2013), but the role of specific synapses at this compartment remained elusive. The only interneuron subtype presently described that innervates the AIS are AACs or chandelier cells (Bazelot et al, 2015), and their inputs to the AIS were selectively targeted by NF knockdown in our study. The resulting impairment in extinction of auditory-cued fear memory adds new insights into the role of such AAC inputs in behavior, even if the integrity of the AIS structure itself is intact.

\section{CONCLUSION}

This model of NF knockdown adds to our understanding of the mechanisms by which specific local GABAergic interneurons contribute to extinction of fear memory. Considering the importance of extinction deficits as a hallmark of anxiety disorders such as phobias and post-traumatic stress disorder, a better understanding of region-specific modification of GABAergic synapses may provide novel and more specific targets for therapeutic intervention.

\section{FUNDING AND DISCLOSURE}

This research was supported by the Werner Reichardt Centre for Integrative Neuroscience (CIN) at the University of Tuebingen, an Excellence Cluster funded by the Deutsche Forschungsgemeinschaft (DFG) within the framework of the Excellence Initiative (EXC 307, Pool Project 2012-09 to IE, $\mathrm{HV}$ and GRL), and by funds from the Charitable Hertie Foundation (IE). The authors declare no conflict of interest.

\section{ACKNOWLEDGMENTS}

We are thankful to Elhanan Ben Yishay for his technical assistance.

\section{REFERENCES}

Ardi Z, Albrecht A, Richter-Levin A, Saha R, Richter-Levin G (2016). Behavioral profiling as a translational approach in an animal model of posttraumatic stress disorder. Neurobiol Dis $\mathbf{8 8}$ : 139-147.

Bazelot M, Bocchio M, Kasugai Y, Fischer D, Dodson PD, Ferraguti F et al (2015). Hippocampal theta input to the amygdala shapes feedforward inhibition to gate heterosynaptic plasticity. Neuron 87: 1290-1303.

Biedenkapp JC, Rudy JW (2009). Hippocampal and extrahippocampal systems compete for control of contextual fear: role of ventral subiculum and amygdala. Learn Mem 16: 38-45.

Bienvenu TCM, Busti D, Magill PJ, Ferraguti F, Capogna M (2012). Cell-type-specific recruitment of amygdala interneurons to hippocampal theta rhythm and noxious stimuli in vivo. Neuron 74: 1059-1074.

Burkarth N, Kriebel M, Kranz EU, Volkmer H (2007). Neurofascin regulates the formation of gephyrin clusters and their subsequent translocation to the axon hillock of hippocampal neurons. $\mathrm{Mol}$ Cell Neurosci 36: 59-70.

Canteras NS, Swanson LW, Angeles L (1992). Projections of the ventral subiculum to the amygdala, septum, and hypothalamus : 
a PHAL Anterograde Tract-Tracing Study in the rat. J Comp Neurol 194: 180-194.

Chaudhry FA, Reimer RJ, Bellocchio EE, Danbolt NC, Osen KK, Edwards RH et al (1998). The vesicular GABA transporter, VGAT, localizes to synaptic vesicles in sets of glycinergic as well as GABAergic neurons. J Neurosci 18: 9733-9750.

Chhatwal JP, Myers KM, Ressler KJ, Davis M (2005). Regulation of gephyrin and GABAA receptor binding within the amygdala after fear acquisition and extinction. J Neurosci 25: 502-506.

Clark BA, Monsivais P, Branco T, London M, Häusser M (2005). The site of action potential initiation in cerebellar Purkinje neurons. Nat Neurosci 8: 137-139.

Cohen AS, Coussens CM, Raymond CR, Abraham WC (1999). Long-lasting increase in cellular excitability associated with the priming of LTP induction in rat hippocampus. J Neurophysiol 82: 3139-3148.

Craig AM, Banker G, Chang W, McGrath ME, Serpinskaya AS (1996). Clustering of gephyrin at GABAergic but not glutamatergic synapses in cultured rat hippocampal neurons. $J$ Neurosci 16: 3166-3177.

Dalton GL, Wang YT, Floresco SB, Phillips AG (2008). Disruption of AMPA receptor endocytosis impairs the extinction, but not acquisition of learned fear. Neuropsychopharmacology 33: 2416-2426.

Dittgen T, Nimmerjahn A, Komai S, Licznerski P, Waters J, Margrie TW et al (2004). Lentivirus-based genetic manipulations of cortical neurons and their optical and electrophysiological monitoring in vivo. Proc Natl Acad Sci USA 101: 18206-18211.

Douglas RJ, Martin KAC (1990). Control of neuronal output by inhibition at the axon initial segment. J Neural Comput 2: 283-292.

Ehrlich I, Humeau Y, Grenier F, Ciocchi S, Herry C, Lüthi A (2009). Amygdala inhibitory circuits and the control of fear memory. Neuron 62: 757-771.

Pape HC, Pare D (2010). Plastic synaptic networks of the amygdala for the acquisition, expression, and extinction of conditioned fear. Physiol Rev 90: 419-463.

Heldt SA, Ressler KJ (2007). Training-induced changes in the expression of GABAA- associated genes in the amygdala after the acquisition and extinction of Pavlovian fear. Eur J Neurosci 26: 3631-3644.

Herry C, Ciocchi S, Senn V, Demmou L, Müller C, Lüthi A (2008). Switching on and off fear by distinct neuronal circuits. Nature 454: 600-606.

Hobin J a., Ji J, Maren S (2006). Ventral hippocampal muscimol disrupts context-specific fear memory retrieval after extinction in rats. Hippocampus 16: 174-182.

Horovitz O, Richter-Levin G (2015). Dorsal periaqueductal gray simultaneously modulates ventral subiculum induced-plasticity in the basolateral amygdala and the nucleus accumbens. Front Behav Neurosci 9: 53.

Howard A, Tamas G, Soltesz I (2005). Lighting the chandelier: new vistas for axo-axonic cells. Trends Neurosci 28: 310-316.

Hübner C, Bosch D, Gall A, Lüthi A, Ehrlich I (2014). Ex vivo dissection of optogenetically activated $\mathrm{mPFC}$ and hippocampal inputs to neurons in the basolateral amygdala: implications for fear and emotional memory. Front Behav Neurosci 8: 64.

Isaacson JS, Scanziani M (2011). How inhibition shapes cortical activity. Neuron 72: 231-243.

Kaphzan H, Buffington S, Ramaraj A, Lingrel J, Rasband M, Santini E et al (2013). Genetic reduction of the $\alpha 1$ Subunit of Na/ $\mathrm{K}$-ATPase corrects multiple hippocampal phenotypes in angelman syndrome. Cell Rep 4: 405-412.

Kim EJ, Horovitz O, Pellman B a, Tan LM, Li Q, Richter-Levin G et al (2013). Dorsal periaqueductal gray-amygdala pathway conveys both innate and learned fear responses in rats. Proc Natl Acad Sci USA 110: 14795-14800.
De Koninck Y, Mody I (1994). Noise analysis of miniature IPSCs in adult rat brain slices: properties and modulation of synaptic GABAA receptor channels. $J$ Neurophysiol 71: 1318-1335.

Kriebel M, Metzger J, Trinks S, Chugh D, Harvey RJ, Harvey K et al (2011). The cell adhesion molecule neurofascin stabilizes axoaxonic GABAergic terminals at the axon initial segment. J Biol Chem 286: 24385-24393.

Kriebel M, Wuchter J, Trinks S, Volkmer H (2012). Neurofascin: a switch between neuronal plasticity and stability. Int J Biochem Cell Biol 44: 694-697.

Lee S, Kim S-J, Kwon O-B, Lee JH, Kim J-H (2013). Inhibitory networks of the amygdala for emotional memory. Front Neural Circuits 7: 129.

Leitermann RJ, Rostkowski AB, Urban JH (2016). Neuropeptide Y input to the rat basolateral amygdala complex and modulation by conditioned fear. J Comp Neurol 524: 2418-2439.

Lesting J, Narayanan RT, Kluge C, Sangha S, Seidenbecher T, Pape H-C (2011). Patterns of coupled theta activity in amygdalahippocampal-prefrontal cortical circuits during fear extinction. PLoS One 6: e21714.

Li XF, Armony JL, LeDoux JE (1996). GABAA and GABAB receptors differentially regulate synaptic transmission in the auditory thalamo-amygdala pathway: an in vivo microiontophoretic study and a model. Synapse 24: 115-124.

Lin H-C, Mao S-C, Gean P-W (2009). Block of gammaaminobutyric acid-A receptor insertion in the amygdala impairs extinction of conditioned fear. Biol Psychiatry 66: 665-673.

Maren S, Fanselow MS, Angeles L (1995). Synaptic plasticity in the basolateral amygdala induced by hippocampal formation stimulation in vivo. J Neurosci 15: 7548-7564.

Maroun M, Richter-Levin G (2002). Local circuit plasticity in the rat dentate gyrus: characterization and aging-related impairment. Neuroscience 112: 1001-1007.

Mascagni F, McDonald AJ (2003). Immunohistochemical characterization of cholecystokinin containing neurons in the rat basolateral amygdala. Brain Res 976: 171-184.

McDonald AJ, Muller JF, Mascagni F (2011). Postsynaptic targets of GABAergic basal forebrain projections to the basolateral amygdala. Neuroscience 183: 144-159.

McGaugh JL (2004). The amygdala modulates the consolidation of memories of emotionally arousing experiences. Annu Rev Neurosci 27: 1-28.

Mello LEAM, Tan AM, Finch DM (1992). GABAergic synaptic transmission in projections from the basal forebrain and hippocampal formation to the amygdala: an in vivo iontophoretic study. Brain Res 587: 41-48.

Milad MRR, Quirk GJJ (2002). Neurons in medial prefrontal cortex signal memory for fear extinction. Nature 420: 70-74.

Otis TS, De Koninck Y, Mody I (1994). Lasting potentiation of inhibition is associated with an increased number of gammaaminobutyric acid type A receptors activated during miniature inhibitory postsynaptic currents. Proc Natl Acad Sci USA 91: 7698-7702.

Pape H-C, Pare D (2010). Plastic synaptic networks of the amygdala for the acquisition, expression, and extinction of conditioned fear. Physiol Rev 90: 419-463.

Quirk GJ, Mueller D (2008). Neural mechanisms of extinction learning and retrieval. Neuropsychopharmacology 33: 56-72.

Seidenbecher T, Laxmi TR, Stork O, Pape H-C (2003). Amygdalar and hippocampal theta rhythm synchronization during fear memory retrieval. Science 301: 846-850.

Somogyi P, Freund TF, Cowey A (1982). The axo-axonic interneuron in the cerebral cortex of the rat, cat and monkey. Neuroscience 7: 2577-2607.

Spampanato J, Polepalli J, Sah P (2011). Interneurons in the basolateral amygdala. Neuropharmacology 60: 765-773. 
Trouche S, Sasaki JM, Tu T, Reijmers LG (2013). Fear extinction causes target-specific remodeling of perisomatic inhibitory synapses. Neuron 80: 1054-1065.

Unal G, Paré J-F, Smith Y, Paré D (2014). Cortical inputs innervate calbindin-immunoreactive interneurons of the rat basolateral amygdaloid complex. J Comp Neurol 522: 1915-1928.

Veres JM, Nagy A, Vereczki K, Andra T, Ha N (2014). Strategically positioned inhibitory synapses of axo-axonic cells potently control principal neuron spiking in the basolateral amygdala. J Neurosci 34: 16194-16206.

Vouimba R-M, Maroun M (2011). Learning-induced changes in mPFC-BLA connections after fear conditioning, extinction, and reinstatement of fear. Neuropsychopharmacology 36: 2276-2285.

Wefelmeyer W, Cattaert D, Burrone J (2015). Activity-dependent mismatch between axo-axonic synapses and the axon initial segment controls neuronal output. Proc Natl Acad Sci USA 112: 9757-9762.

Wolff SBE, Gründemann J, Tovote P, Krabbe S, Jacobson G a, Müller C et al (2014). Amygdala interneuron subtypes control fear learning through disinhibition. Nature 509: 453-458.

Woodruff AR, Anderson SA, Yuste R (2010). The enigmatic function of chandelier cells. Front Neurosci 4: 1-11.
Zitman FMP, Lucas M, Trinks S, Grosse-Ophoff L, Kriebel M, Volkmer H et al (2014). Dentate gyrus local circuit is implicated in learning under stress-a role for neurofascin. Mol Neurobiol 53: $842-850$.

Zonta B, Desmazieres A, Rinaldi A, Tait S, Sherman DL, Nolan MF et al (2011). A critical role for neurofascin in regulating action potential initiation through maintenance of the axon initial segment. Neuron 69: 945-956.

(i) (2) This work is licensed under a Creative Commons Attribution-NonCommercial-ShareAlike 4.0 International License. The images or other third party material in this article are included in the article's Creative Commons license, unless indicated otherwise in the credit line; if the material is not included under the Creative Commons license, users will need to obtain permission from the license holder to reproduce the material. To view a copy of this license, visit http://creativecommons.org/licenses/by-nc-sa/4.0/

(C) The Author(s) 2017

Supplementary Information accompanies the paper on the Neuropsychopharmacology website (http://www.nature.com/npp) 